\title{
Modelo experimental para estudo de desenluvamento cutâneo no membro inferior
}

\author{
Experimental model of degloving injury in hind limb
}

\author{
Dimas Andre Milcheski ${ }^{1}$ \\ Marcus Castro Ferreira ${ }^{2}$ \\ Paulo Tuma JR. ${ }^{1}$
}

Hugo Alberto NaKamoto ${ }^{3}$

Trabalho realizado no Laboratório de Microcirurgia (LIM/04) da Disciplina de Cirurgia Plástica da Faculdade de Medicina da Universidade de São Paulo, São Paulo, SP, Brasil.

Artigo submetido no SGP (Sistema de Gestão de Publicações) da RBCP.

Artigo recebido: 29/7/2010 Artigo aceito: 18/10/2010

\begin{abstract}
RESUMO
Os desenluvamentos cutâneos são lesões graves e frequentemente há dificuldade para o cirurgião decidir qual o tratamento mais adequado a ser instituído, o reposicionamento e a sutura do retalho ou o desengorduramento do retalho e a enxertia da pele avulsionada. A conduta cirúrgica de reposicionamento e sutura do retalho é mais rápida e simples de ser realizada, mantém as características anatômicas e fisiológicas locais, mas frequentemente evolui com perda parcial ou total do retalho avulsionado. $\mathrm{O}$ tratamento cirúrgico de adelgaçamento do retalho desenluvado e enxertia da pele obtida tem a desvantagem de resultar em aspecto estético e protetivo inferiores, mas é o tratamento mais utilizado, devido à maior taxa de integração do enxerto. Medicações com propriedades de aumentar a perfusão do retalho desenluvado podem permitir a conduta cirúrgica de reposicionamento e sutura do retalho ao seu leito original, mantendo as vantagens da cobertura cutânea original e preservando total ou parcialmente a viabilidade do retalho. Para tanto, há necessidade de se desenvolver um modelo experimental para testar medicações com propriedades vasculares. O presente estudo apresenta um modelo animal de desenluvamento cutâneo em membros inferiores de ratos. Os animais foram submetidos ao modelo proposto de desenluvamento de todo membro inferior, resultando em um retalho de fluxo distal, que foi reposicionado ao leito e suturado.
\end{abstract}

Descritores: Ferimentos e lesões. Extremidade inferior. Pele/lesões. Necrose/etiologia. Modelos animais.

\begin{abstract}
Degloving injuries may be a challenge when it comes to deciding the surgical approach to be used. Repositioning of the flap and suturing is faster and more straightforward, but often leads to total or partial loss of the avulsed flap. Skin flap deffating and grafting of the detached flap have the disadvantages of resulting in poor aesthetic appearance and being less protective, but they have been the most widely used due to the higher rate of graft take. Pharmacological agents with vascular properties that enhance the viability of the reattached flap could be beneficial to patients with degloving injuries. In a way to test drugs with vascular properties is necessary to develop an experimental model. This paper presents an animal model of degloving injury in the hind limb of rats. Rats were subjected to the proposed degloving model of hind limb, resulting in a reverse flow flap. The flap was then repositioned and sutured.
\end{abstract}

Keywords: Wounds and injuries. Lower extremity. Skin/injuries. Necrosis/etiology. Animal models.

1. Doutor em Cirurgia Plástica pela Faculdade de Medicina da Universidade de São Paulo (FMUSP); Médico Assistente da Divisão Cirurgia Plástica do Hospital das Clínicas da FMUSP (HC-FMUSP), São Paulo, SP, Brasil.

2. Professor Titular da Disciplina de Cirurgia Plástica da FMUSP, São Paulo, SP, Brasil.

3. Mestre em Cirurgia Plástica pela Faculdade de Medicina da Universidade de São Paulo (FMUSP); Médico Assistente da Divisão Cirurgia Plástica do Hospital das Clínicas da FMUSP (HC-FMUSP), São Paulo, SP, Brasil. 


\section{INTRODUÇÃO}

Os desenluvamentos nos membros inferiores são, frequentemente, lesões graves e apresentam dificuldade para decidir qual o tratamento cirúrgico mais adequado a ser instituído ${ }^{1}$.

Os desenluvamentos também são chamados de ferimentos descolantes ou de avulsões parciais. O termo desenluvamento é tradução da palavra inglesa degloving e é o mais citado na literatura inglesa. Consideramos os três termos equivalentes.

Observa-se, atualmente, aumento da incidência de ferimentos descolantes. No Hospital das Clínicas da Faculdade de Medicina da Universidade de São Paulo (HC-FMUSP), temos observado, nos últimos anos, aumento significativo da incidência dos desenluvamentos em membros inferiores, geralmente associados a traumatismos graves e extensos, relacionados a atropelamentos ou a acidentes entre motociclistas e automóveis ${ }^{2}$.

As lesões são secundárias à preensão da extremidade entre uma superfície móvel e uma superfície fixa, como ocorre nos casos de atropelamentos ${ }^{3}$. A pele e o subcutâneo desenluvados ficam conectados apenas por uma das extremidades do membro. Se não houver avaliação adequada e tratamento precoce, pode ocorrer insuficiência circulatória, seja arterial ou venosa, com consequente necrose do tecido descolado.

Há grande dificuldade no tratamento destes pacientes, devido à extensão do ferimento descolante e à presença de traumatismos associados, com risco de perda da extremidade. $\mathrm{O}$ aumento do interesse nas avulsões parciais em membros inferiores motivou a elaboração de um protocolo para padronização do atendimento destes pacientes no Pronto Socorro do HC-FMUSP pela Disciplina de Cirurgia Plástica através de seu grupo de Feridas Complexas, com a finalidade de diminuir a morbidade, normatizar o atendimento e obter melhor evolução dos pacientes atendidos ${ }^{4,5}$.

Há duas condutas cirúrgicas para o tratamento dos ferimentos descolantes. A primeira consiste na limpeza da ferida e reposicionamento do retalho a sua posição original e, a segunda, na limpeza da ferida e ressecção do retalho desenluvado, com obtenção de enxerto de pele parcial a partir do retalho descolado, seguida por enxertia no atendimento inicial. O sucesso do tratamento depende de alguns fatores, tais como o tempo decorrido do trauma (maior ou menor que 24 horas), a presença de instabilidade hemodinâmica e a viabilidade do retalho avulsionado ${ }^{4,5}$.

A conduta cirúrgica de reposicionamento do retalho, a princípio mais rápida e direta, pode resultar em necrose parcial ou total do retalho desenluvado. Ao se realizar o reposicionamento e sutura do retalho avulsionado a sua posição original, fármacos que aumentem a viabilidade do retalho descolado poderiam ser utilizados no intuito de diminuir a área de necrose deste retalho. Há pouca referência a modelos experimentais de desenluvamentos na literatura ${ }^{6-8}$. Nenhum destes estudos se refere a lesões em membros inferiores e apenas o modelo proposto por Oztuna et al. ${ }^{7}$ testa medicação para aumento da viabilidade do retalho avulsionado.

Os autores apresentam modelo experimental de desenluvamento do tegumento cutâneo em membros inferiores de ratos, desenvolvido no Laboratório de Microcirurgia (LIM/04) da Faculdade de Medicina da Universidade de São Paulo.

Esse estudo em desenvolvimento permite testar medicações com a finalidade de diminuir a área de necrose do retalho desenluvado e ressuturado.

\section{MÉTODO}

O modelo apresentado é parte de projeto experimental para avaliação de medidas terapêuticas para o desenluvamento em membro inferior de ratos e foi aprovado pela Comissão de Projetos de Pesquisa do HC-FMUSP, sob o número 0842/09.

$\mathrm{O}$ estudo segue as orientações das entidades envolvidas na condução de pesquisas experimentais (Council for International Organization of Medical Sciences / Ethical Code for Animal Experimentation / Colégio Brasileiro de Experimentação Animal).

O modelo utiliza ratos da linhagem Wistar, pesando entre 200 e 350 gramas. Após a anestesia (tiopental sódigo; $40 \mathrm{mg} /$ $\mathrm{kg}$ ), realiza-se tricotomia da extremidade inferior e o animal é colocado em posição operatória.

\section{Procedimento cirúrgico}

Procede-se à incisão circunferencial na base da coxa, conforme marcação prévia, abrangendo pele e subcutâneo (Figura 1).

Quatro ganchos são posicionados (quadrantes anterior, posterior, medial e lateral) e realiza-se tração uniforme com força moderada, levando ao desenluvamento do retalho até a região do tornozelo, na altura do terço distal da perna do animal (Figura 2).

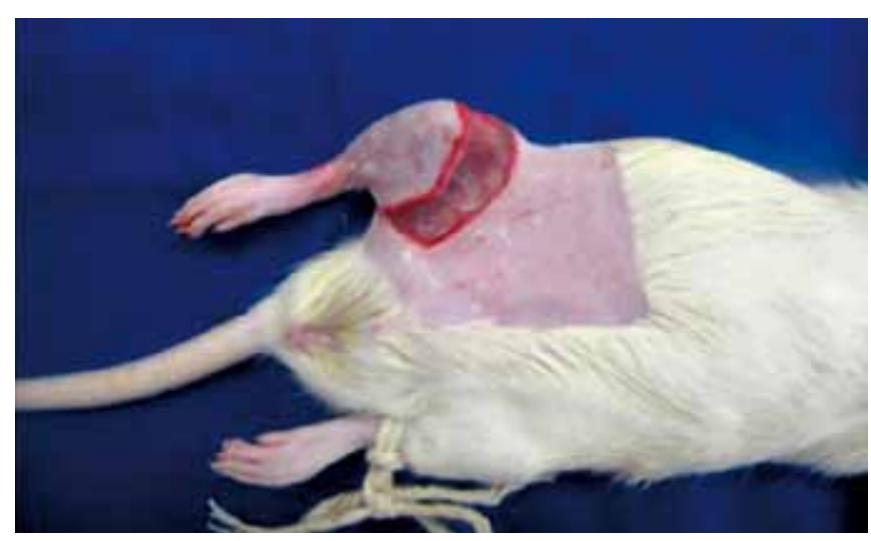

Figura 1 - Incisão circunferencial da pele e do subcutâneo. 


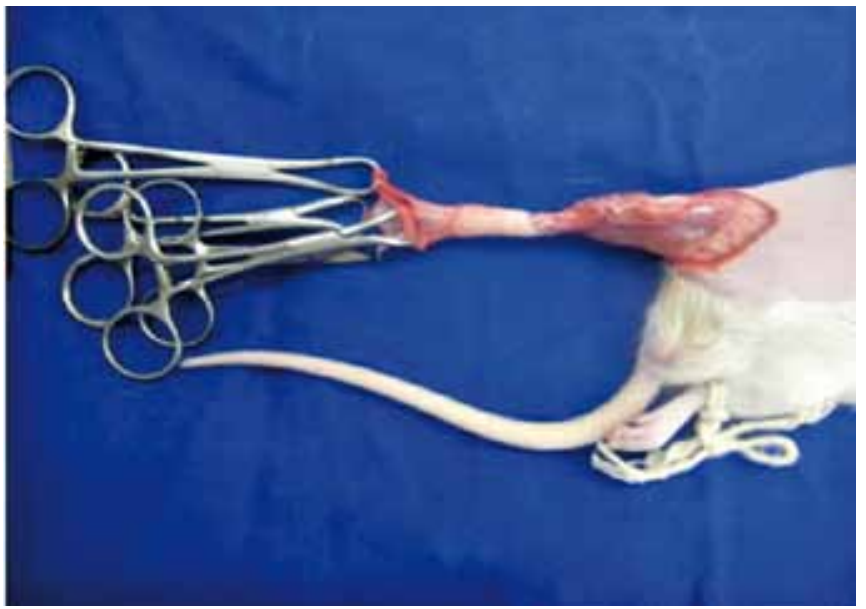

Figura 2 - Desenluvamento do retalho até a porção distal da perna.

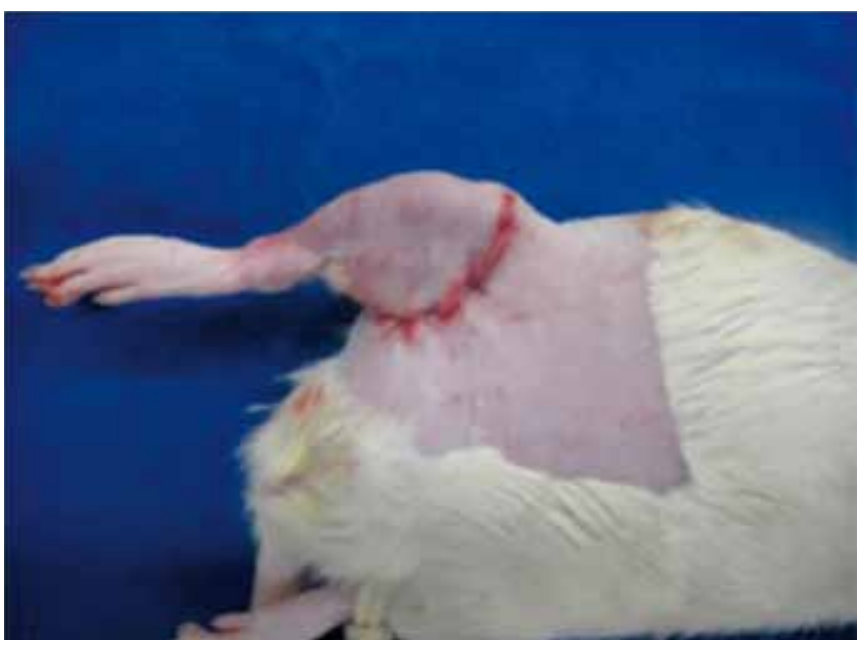

Figura 3 - Sutura contínua da pele com nylon 5.0.

Após 5 minutos, realiza-se reposicionamento do retalho ao seu leito original e sutura da pele (nylon 5.0 contínuo) (Figura 3 ).

Finalmente, acondiciona-se o animal em recipiente individual com água e ração ad libitum e período claro/escuro 12/12 horas.

\section{Pós-operatório}

O animal é observado diariamente para verificação de sinais de viabilidade ou inviabilidade do retalho avulsionado (coloração da pele, enchimento capilar, isquemia, congestão ou necrose), e presença de complicações cirúrgicas (hematoma, deiscência de ferida e infecção) (Figura 4).

Os animais são sacrificados no $7^{\circ}$ dia pós-operatório.

\section{Avaliação da área total, área de viabilidade e área de necrose do retalho}

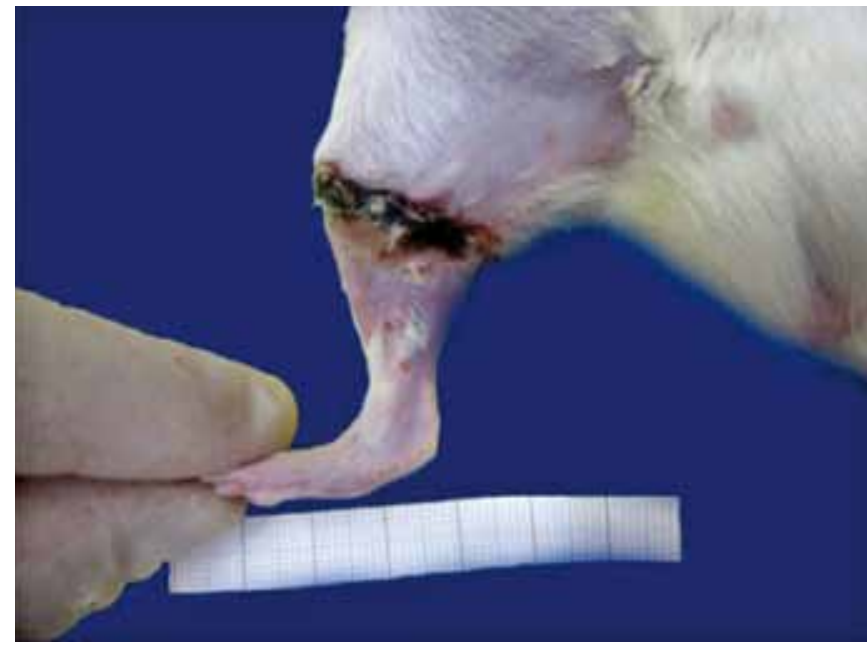

Figura 4 - Necrose parcial do retalho no $7^{\circ}$ dia pós-operatório.

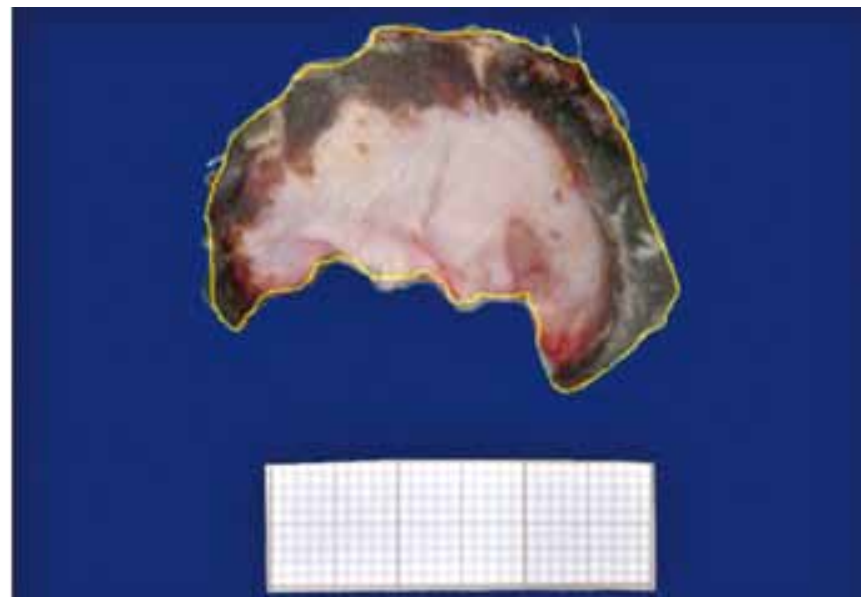

Figura 5-Quantificação da área total do retalho através do programa Image $J$.

Após o sacrifício, realiza-se retirada de todo retalho avulsionado e coloca-se o tecido planificado sobre a mesa operatória com uma escala em centímetros e retira-se foto digital padronizada.

A imagem digital é analisada com auxílio do programa de computador Image $J^{9}$, adequado ao cálculo de áreas, sendo então quantificadas as áreas de necrose, de viabilidade e total do retalho (Figura 5).

\section{REFERÊNCIAS}

1. Mandel M. The management of lower extremity degloving injuries. Ann Plast Surg. 1981;6(1):1-5.

2. Milcheski DA. Análise crítica do tratamento de pacientes com ferimentos descolantes nos membros inferiores [Dissertação]. São Paulo:Faculdade de Medicina, Universidade de São Paulo;2009. 73p. 
3. Kudsk KA, Sheldon GF, Walton RL. Degloving injuries of the extremities and torso. J Trauma. 1981;21(10):835-9.

4. Milcheski DA, Nakamoto HA, Tuma Jr. P, Gemperli R, Ferreira MC. Tratamento cirúrgico de ferimentos descolantes nos membros inferiores: proposta de protocolo de atendimento. Rev Col Bras Cir. 2010;37(3):199-203.

5. Jeng SF, Hsieh CH, Kuo YR, Wei FC. Technical refinement in the management of circumferentially avulsed skin of the leg. Plast Reconstr Surg. 2004;114(5):1225-7.
6. KurataT, O’Brien BM, Black MJ. Microvascular surgery in degloving injuries: an experimental study. Br J Plast Surg. 1978;31(2):117-20.

7. Oztuna V, Eskandari MM, Unal S, Colak M, Karabacak T. The effect of pentoxifylline in treatment of skin degloving injuries: an experimental study. Injury. 2006;37(7):638-41.

8. Wang ZT, Guo SZ, Xiu ZF, Lu KH, Li QS. A new model of skin avulsion injuries in rats. Zhonghua Zheng Xing Wai Ke Za Zhi. 2008;24(3):212-5.

9. Image J 1.42q for Macintosh. Versão 10.2. Wayne Resband National Institutes of Health, USA. Disponível em: http://rsbweb.nih.gov/ij/ download.html.

\section{Correspondência para:}

Dimas Andre Milcheski

Rua Alves Guimarães, 855, apto 54 - Pinheiros - São Paulo, SP, Brasil - CEP 05410-001

E-mail: drdimasandre@gmail.com 